\title{
Carbonated drink consumption and increased exhaled nitric oxide in atopic children
}

\section{To the Editors:}

Fractional exhaled nitric oxide concentrations (FeNO) are elevated in asthmatic children and have been used inter alia to guide inhaled corticosteroid treatment in children [1]. Caffeine consumption might influence FeNO independently of asthma and more than one plausible mechanism has been identified. Studies in adults have reported changes in FeNO within $1 \mathrm{~h}$ of caffeine ingestion but the results are inconsistent, with one study having reported reduced FeNO [2], one elevated FeNO [3] and another no change after ingestion [4].

An association between caffeine intake and altered FeNO is equally plausible in children but has not been sought. The predominant source of caffeine in children's diet is carbonated drinks, in particular colas, and in Scotland "Irn Bru" (a carbonated orange-coloured drink). As part of a longitudinal birth cohort study where diet was related to asthma outcomes, including FeNO [5], we have been able to test the hypothesis that FeNO in 5-yr-old children is increased in association with regular intake of carbonated drinks.

Our methodology is described fully elsewhere [5]. Briefly, parents were asked to complete a respiratory and food frequency questionnaire (FFQ) and they were invited to bring their child to the hospital for an assessment, including FeNO measurement and skin-prick reactivity to four common allergens. Atopy was defined as at least one positive skin-prick test. Drinks were described as either regular fizzy drinks (e.g.

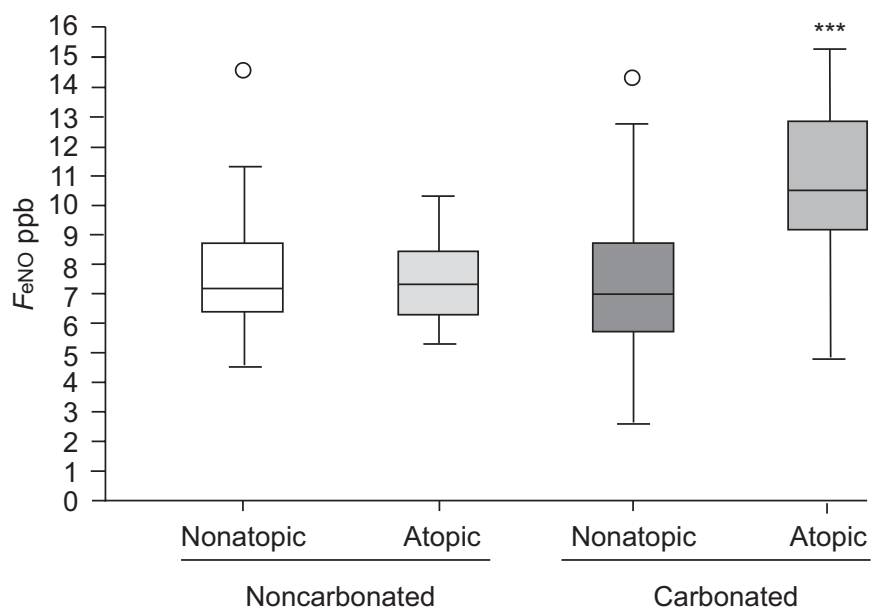

FIGURE 1. A box-whiskers plot demonstrating median and interquartile ranges for fractional exhaled nitric oxide ( $F_{\mathrm{eNO}}$, parts per billion $\left.(\mathrm{ppb})\right)$ across groups categorised by atopy and consumption of carbonated drinks. $\square: n=47 ; \square: n=20$; 口: $n=59 ; \quad \square: n=21 .{ }^{* * *}: \mathrm{p}<0.001$ (ANOVA) for comparisons with other groups lemonade, Irn Bru, cola) or low-calorie fizzy drinks. Children's intake of carbonated drinks at 5 yrs of age was ascertained by parents' responses to one of nine frequency-of-consumption categories ranging between "rarely or never" to "seven or more measures per day". A measure was defined as half a standard can or one medium glass full, equivalent to $165 \mathrm{~mL}$. For the purpose of the present study, carbonated drink consumption ("exposed" or "nonexposed") was defined as at least one measure of carbonated drink per month. A standard chemiluminescence analyser $\left(\mathrm{NIOX}_{\mathbb{R}}\right.$; Aerocrine, Solna, Sweden) was used to measure FeNO at an expiratory flow of $50 \mathrm{~mL} \cdot \mathrm{s}^{-1}$.

Carbonated drinks (regular and/or diet) were consumed by $646(58 \%)$ out of 1,111 children whose parents completed an FFQ. FeNO data were available in 157 children, of whom atopy was determined in 147. The median consumption of carbonated drinks was one per week (interquartile range one per month to two per week). There was a trend, which did not reach significance, for FeNO to be elevated among the 85 children who drank carbonated drinks (geometric mean \pm SEM $6.8 \pm 0.7 \mathrm{ppb}$ ) compared with the remaining 72 children who did not $(6.0 \pm 0.7 \mathrm{ppb} ; \mathrm{p}=0.112$, Mann-Whitney U-test). We stratified by atopy, recognised as a confounder for FeNO. The FeNO values were $5.9 \pm 0.5,5.4 \pm 0.5,6.1 \pm 0.5$ and $11.9 \pm 1.8 \mathrm{ppb}$ in nonexposed nonatopic $(n=47)$, exposed nonatopic $(n=59)$, nonexposed atopic $(n=20)$ and exposed atopic $(n=21)$ children, respectively (fig. 1). The latter was significantly higher $(p<0.001)$ when compared with other groups. Multivariate analysis confirmed this association to be independent of asthma. We sought evidence of a dose-response relationship by dichotomising the 21 exposed atopic children: FeNO was $13.1 \pm 2.6$ and $10.6 \pm 2.4 \mathrm{ppb}$ among the higher and lower exposure groups, respectively.

This exploratory study is the first to seek an association between caffeine intake and fractional exhaled nitric oxide in children. The clinical relevance of this association is not clear. Since approximately two-thirds of asthmatic children are atopic and many children consume caffeine-containing carbonated drinks, an association between fractional exhaled nitric oxide and caffeine in atopic children could be of clinical relevance and might also confound epidemiological studies. An intervention study is needed to study the relationship between fractional exhaled nitric oxide and caffeine intake in atopic children.

S.W. Turner*, L.C.A. Craig", P.J. Harbour*, S.H. Forbes*, S. Martindale ${ }^{\#}$, G. McNeill*,\#, A. Seaton", J.G. Ayres", G. Devereux" and P.J. Helms*

Depts of *Child Health, and "Environmental and Occupational Medicine, University of Aberdeen, Aberdeen, UK. 


\section{STATEMENT OF INTEREST}

None declared.

\section{REFERENCES}

1 Pijnenburg MW, Bakker EM, Hop WC, De Jongste JC. Titrating steroids on exhaled nitric oxide in children with asthma: a randomized controlled trial. Am J Respir Crit Care Med 2005; 172: 831-836.

2 Bruce C, Yates DH, Thomas PS. Caffeine decreases exhaled nitric oxide. Thorax 2002; 57: 361-363.

3 Warke TJ, Shields MD, Finnegan J, Shields MD. Caffeine and exhaled nitric oxide. Thorax 2003; 58: 281.
4 Taylor ES, Smith AD, Cowan JO, Herbison GP, Taylor DR. Effect of caffeine ingestion on exhaled nitric oxide measurements in patients with asthma. Am J Respir Crit Care Med 2004; 169: 1019-1021.

5 Devereux G, Turner SW, Craig LC, et al. Low maternal vitamin $\mathrm{E}$ intake during pregnancy is associated with asthma in 5-year-old children. Am J Respir Crit Care Med 2006; 174: 499-507.

\section{XDR-TB: Indian perspective}

\section{To the Editors:}

I read with interest the recent article by MigLIORI et al. [1] in the March issue of the European Respiratory Journal on the new extensively drug-resistant tuberculosis (XDR-TB) threat, wherein the authors have comprehensively covered the topic and tried to alert the medical fraternity of the need to unite in order to fight this global threat.

There is no doubt that the development of multidrug-resistant (MDR)-TB and XDR-TB reveals weaknesses in primary care diagnostic services and a failure to adhere to the World Health Organization's directly observed treatment, short course (DOTS) strategy.

Although XDR-TB has not been reported from India, I would like to share certain facts pertinent to India. First, TB has been a major public health problem in India. Today, India accounts for nearly one third of the global TB burden. A population of 14 million is estimated to be suffering from TB, of which 3-3.5 million are highly infectious. The incidence rate of the disease is very high, with an estimate of $>2$ million new cases of TB occurring every year [2].

Secondly, most TB patients first seek help from one of India's 10 million private practitioners. It is estimated that for most of these patients it is up to 4-6 weeks before they are diagnosed as having TB. In addition to this, the TB cure rates for patients who remain with private practitioners are low [3].

Thirdly, community awareness and attitudes towards the symptoms of TB and knowledge about the cause of the disease are very low, especially among the needy, and the "TB stigma" continues [4].

Fourthly, there are, at present, only three quality-assured laboratories for culture and drug susceptibility testing in the entire nation. In the absence of the readily available culture and sensitivity services, even clinicians at medical schools are making the diagnosis of MDR-TB based on clinical and radiological grounds and are empirically treating the patients with nonstandardised regimens, thus increasing the prevalence of MDR- and XDR-TB. The worst part is that we do not have access to the culture and sensitivity services to warn the global community about the latest epidemic of XDR-TB.

Fifth, most of the second-line anti-tubercular drugs like fluoroquinolones and aminoglycosides are routinely available over the counter and are misused to treat trivial illnesses, including upper respiratory tract infections, which will increase the overall level of resistance in the community [5].

Finally, the efficacy of the DOTS strategy has been proven beyond doubt; then why are the nations not sticking to this strategy, allowing hundreds of brands and combinations of antitubercular drugs to be freely available over the counter?

\section{A. Singh}

Christian Medical College and Hospital Ludhiana, Ludhiana, India.

\section{STATEMENT OF INTEREST}

None declared.

\section{REFERENCES}

1 Migliori GB, Loddenkemper R, Blasi F, Raviglione MC. 125 years after Robert Koch's discovery of the tubercle bacillus: the new XDR-TB threat. Is "science" enough to tackle the epidemic? Eur Respir J 2007; 29: 423-427.

2 Revised National Tuberculosis Control Programme, TB India. TB India 2006 RNTCP Status Report. www.tbcindia. org/pdfs / Annual\%20Report\%20TB\%202006.pdf. Date last accessed: March 1, 2007.

3 World Health Organization, Regional Office for South-East Asia. Involving Private Medical Practitioners in TB and STI Control: Experiences in Public-Private Partnerships. www.searo.who.int/en/Section10/Section18/Section356/ Section413_1889.htm. Date last accessed: March 1, 2007. Date last updated: April 2006. 